\title{
Conductividad térmica y comportamiento mecánico del hormigón en las estructuras termoactivas. Thermal conductivity and mechanical behavior of concrete in thermoactive structures.
}

\author{
Teresa Romanos Blázquez, Alfonso Cobo Escamilla \\ Universidad Politécnica de Madrid (Spain, t.rblazquez@alumnos.upm.es, alfonso.cobo@upm.es)
}

\begin{abstract}
The aim of this project is to develop and study different kinds of concrete in order to improve its thermal properties to be used in reinforced concrete structures of thermally active building systems (TABS). For this reason different specimens and testing procedures were proposed to study, on the one hand thermal conductivity of concrete made with three different types of cement (CEM I, CEM II and CEM III) and two different kinds of aggregate (limestone and siliceous) at three different levels of humidity $(0 \%, 50 \%$ and $100 \%)$ was analized. On the other hand, compressive strength of concrete was also studied. Results from thermal tests showed that specimens made of concrete with cement CEM II, siliceous aggregate and humidity of $100 \%$ had higher values of conductivity than the other specimens; while mechanical tests showed that concrete with cement CEM I and limestone aggregate had a higher compressive strength. In conclusion, concrete made of cement type CEM II and siliceous aggregate improved the conductivity of concrete for its use on thermal active structures. Thermal conductivity was also improved by high levels of humidity in concrete. Contrary to the expectations, concrete specimens made of cement CEM III didn't show higher thermal conductivity levels than concrete made of cement CEM I and CEM II.
\end{abstract}

Index Terms - Concrete; thermally active structures; thermal conductivity; compressive strength.

Resumen - El objetivo del trabajo es el análisis de la influencia de distintas variables sobre la conductividad térmica del hormigón y su resistencia a compresión, para ser usado en estructuras termoactivas. Se valoró la influencia del tipo de cemento, el tipo de árido y la humedad del hormigón. Se llevó a cabo una campaña experimental de probetas de hormigón H-25, elaboradas con tres tipos de cemento diferentes (CEM I, CEM II y CEM III) combinados con dos tipos de árido (calizo y silíceo) en el que se estudió por una parte la conductividad térmica del hormigón en tres grados de humedad diferentes $(0 \%, 50 \%$ y $100 \%$ de humedad) y por otra parte la resistencia a compresión del hormigón. Los resultados de los ensayos térmicos mostraron valores de conductividad superiores en las muestras elaboradas con cemento CEM II, árido silíceo y $100 \%$ de humedad. Los resultados derivados de los ensayos a compresión indicaron que las muestras con cemento del tipo CEM I y árido calizo ofrecieron valores de resistencia a compresión superiores a las del resto de las muestras. Contrariamente a lo que se esperaba, las muestras de hormigón elaboradas con cemento CEM III no ofrecieron valores de conductividad superiores al resto de muestras. Se puede concluir que el hormigón elaborado con cemento CEM II y árido silíceo es más adecuado para su empleo en los elementos estructurales de hormigón de las estructuras termoactivas, siempre que los requerimientos estructurales no se vean comprometidos.

Palabras clave-Cemento; estructuras termoactivas; conductividad térmica; compresión.

\section{INTRODUCCIÓN}

$\mathrm{E}$ L calentamiento global y el deterioro del planeta Tierra son asuntos que ocupan los esfuerzos de los distintos países para llegar a una serie de acuerdos que promuevan la conservación del medio ambiente.

Los cambios en el clima, y sus efectos adversos son una preocupación común de toda la humanidad. Las actividades de 
la población mundial han ido aumentando sustancialmente las concentraciones de gases de efecto invernadero en la atmósfera, produciendo un calentamiento adicional de la superficie y la atmósfera de la Tierra (Naciones Unidas, 1992).

La mayoría de la energía empleada para climatizar los edificios se consume para mantener temperaturas interiores en torno a los $20^{\circ} \mathrm{C}$. Los sistemas energéticos de baja exergía se fundamentan en el empleo de temperaturas moderadas tanto en calefacción como en refrigeración, lo cual permite y favorece el empleo de fuentes de energía renovables en los edificios, reduciendo considerablemente el empleo de combustibles fósiles (Torio \& Schmidt, 2011).

Las estructuras termoactivas son aquellas que emplean la inercia térmica del hormigón de las estructuras para el intercambio de energía térmica en un edificio formando parte de su sistema de climatización. A través de un circuito cerrado y estanco de conductos de polietileno de alta densidad embebidos en la estructura de hormigón, circula un fluido caloportador para llevar a cabo la climatización del edificio (Ortega, 2014). De este modo, en un sistema de captación de energía geotérmica, las estructuras de hormigón termoactivas pueden formar parte de la cimentación de los edificios como intercambiadores de calor subterráneo, y las estructuras de hormigón interiores del edificio pueden constituir el sistema de climatización que distribuye el calor o el frío hacia las distintas estancias (Brandl, 2006).

Los principales beneficios de este sistema pasan por, entre otros, el empleo de energías renovables al emplear rangos de temperaturas moderadas (Lehmann, Dorer \& Koschenz, 2007).

Las variables que se han abordado en este estudio para el análisis del comportamiento del hormigón en las estructuras termoactivas han sido la conductividad térmica del hormigón y su resistencia a compresión.

Según estudios como el de Zoldners (Zoldners, 1973) la conductividad térmica del hormigón depende, en su mayor parte, de la conductividad del árido, de la conductividad de la pasta de cemento, de la dosificación de la mezcla de sus componentes, de la compacidad y del grado de humedad del hormigón. En investigaciones, como la de Naus (Naus, 2010) se concluyó que los factores primarios que determinan la conductividad térmica del hormigón son su contenido de humedad, el volumen de poro y su distribución, y la cantidad y tipo de árido.

En otros estudios (Svanholm, 1980) se determinó que la conductividad térmica del hormigón se ve altamente condicionada por su densidad y su grado de porosidad, comprobando que los hormigones aligerados, con un elevado grado de porosidad, presentan bajas conductividades térmicas.

La mayoría de los estudios existentes sobre el comportamiento térmico del hormigón endurecido se centran en cómo le afectan las altas temperaturas (superiores a $200^{\circ} \mathrm{C}$ ). En los estudios realizados por Arioz (Arioz, 2007) no se observaron cambios superficiales en el hormigón analizado hasta que no se alcanzaron $\operatorname{los} 400^{\circ} \mathrm{C}$ de temperatura.

Atendiendo al análisis del comportamiento mecánico del hormigón, encontramos investigaciones en las que se comprueba que varía en función de la tempertura. Existen estudios (Naus, 2010) que determinan que con el aumento de la temperatura entre $\operatorname{los} 22^{\circ} \mathrm{C}$ y los $120^{\circ} \mathrm{C}$, el hormigón sufre una disminución en su resistencia a compresión. De acuerdo con este estudio, el tipo de árido empleado en la elaboración del hormigón condiciona su resistencia a compresión a altas temperaturas. Las muestras de hormigón con árido silíceo ofrecieron resistencias inferiores a las muestras de hormigones realizados con árido calizo y hormigón ligero.

En estudios como el de Youssef (Youssef \& Moftah, 2007) se analizó el comportamiento mecánico del hormigón sometido a elevadas temperaturas, durante incendios, y se determinó que la resistencia del hormigón y el módulo de deformación inicial del hormigón decrecían, mientras que el valor de la deformación máxima del hormigón aumentaba.

En otras investigaciones se comprobó que los factores más determinantes en la deformación del hormigón sometido a elevadas temperaturas son las tensiones de colapso térmico y la presión del poro, determinante en la permeabilidad del hormigón (Fu \& Li, 2010).

\section{DISPOSITIVO EXPERIMENTAL}

\section{A. Materiales empleados}

Para llevar a cabo la parte experimental del trabajo se emplearon los materiales que se indican a continuación, con las dosificaciones que se muestran en la tabla 1:

- La arena que se empleó fue un árido fino de fracción granulométrica 0-4 mm, de naturaleza silícea, lavada según la norma UNE-EN 197- 1:2011 y RC-16.

- La grava empleada en las amasadas 1, 3 y 5 fue un árido grueso de granulometría 4-20 mm de naturaleza silícea, lavada según la norma UNE-EN 12620:2003+A1:2009.

- La grava empleada en las amasadas 2, 4 y 6 fue un árido grueso de granulometría 4-20 mm de naturaleza caliza, lavada según la norma UNE-EN 12620:2003+A1:2009.

- Se empleó cemento de tres tipos distintos. Todos ellos cumpliendo con la Instrucción RC-16 para la recepción de cementos, y elaborados conforme a la norma UNE- EN 197$1: 2011$.

- Amasadas 1 y 2: CEM II/B-L 32,5 N.

- Amasadas 3 y 4: CEM I 52,5 N-SR 5.

- Amasadas 5 y 6: CEM III/B 42,5.

- Se empleó agua procedente del suministro del Canal de Isabel II. 
Como se observa en la tabla 1, se combinaron tres tipos de cemento (CEM I, CEM II y CEM III) con dos tipos de grava distintos (grava de origen silíceo y grava de origen calizo) respectivamente en las seis amasadas, empleando la misma dosificación en sus componentes para todas las amasadas.

TABLA I

DOSIFICACIONES Y TIPOS DE COMPONENTES DE CADA AMASADA

\begin{tabular}{|c|c|c|c|c|c|c|}
\hline Amasada & 1 & 2 & 3 & 4 & 5 & 6 \\
\hline $\begin{array}{l}\text { Tipo de } \\
\text { cemento }\end{array}$ & $\begin{array}{c}\text { CEM } \\
\text { II/ B-L } \\
32,5 \mathrm{~N}\end{array}$ & $\begin{array}{c}\text { CEM } \\
\text { II/ B-L } \\
32,5 \mathrm{~N}\end{array}$ & $\begin{array}{c}\text { CEM I } \\
52,5 \\
\text { N-SR } 5\end{array}$ & $\begin{array}{c}\text { CEM I } \\
52,5 \\
\text { N-SR } 5\end{array}$ & $\begin{array}{l}\text { CEM } \\
\text { III/B } \\
42,5\end{array}$ & $\begin{array}{l}\text { CEM } \\
\text { III/B } \\
42,5\end{array}$ \\
\hline $\begin{array}{l}\text { Tipo de } \\
\text { grava }\end{array}$ & $\begin{array}{l}\text { Árido } \\
\text { calizo }\end{array}$ & $\begin{array}{l}\text { Árido } \\
\text { silíceo }\end{array}$ & $\begin{array}{l}\text { Árido } \\
\text { calizo }\end{array}$ & $\begin{array}{l}\text { Árido } \\
\text { silíceo }\end{array}$ & $\begin{array}{l}\text { Árido } \\
\text { calizo }\end{array}$ & $\begin{array}{l}\text { Árido } \\
\text { silíceo }\end{array}$ \\
\hline $\begin{array}{c}\text { Cemento } \\
(\mathrm{kg})\end{array}$ & 7,20 & 7,20 & 7,20 & 7,20 & 7,20 & 7,20 \\
\hline $\begin{array}{c}\text { Arena } \\
(\mathrm{kg})\end{array}$ & 15,00 & 15,00 & 15,00 & 15.00 & 15,00 & 15,00 \\
\hline $\begin{array}{c}\text { Grava } \\
(\mathrm{kg})\end{array}$ & 26,00 & 26,00 & 26,00 & 26,00 & 26,00 & 26,00 \\
\hline $\begin{array}{c}\text { Agua } \\
(\mathrm{kg})\end{array}$ & 3.80 & 4,00 & 3,73 & 3,80 & 4,00 & 3,55 \\
\hline
\end{tabular}

\section{B. Fases del trabajo}

Para evaluar el comportamiento térmico y mecánico del hormigón se realizaron ensayos en el laboratorio de materiales en los que se midieron los valores de conductividad térmica en diferentes probetas de hormigón, y a su vez se sometieron a ensayos destructivos de compresión.

Para analizar la conductividad térmica del hormigón, se elaboraron noventa probetas cúbicas, las cuales fueron sometidas a diferentes grados de humedad: $0 \%$ de humedad, $50 \%$ de humedad y $100 \%$ de humedad, para determinar su influencia sobre la conductividad térmica.

Para la evaluación de la resistencia mecánica a compresión del hormigón se elaboraron dieciocho probetas cilíndricas en total, que fueron sometidas a ensayos destructivos a compresión sobre prensa hidráulica para determinar la resistencia del hormigón en función del tipo de árido y cemento empleados.

\section{Proceso experimental}

En la elaboración de las seis amasadas de hormigón se empleó una amasadora planetaria de eje vertical. Para realizar las muestras de hormigón se procedió a rellenar los moldes de las probetas cúbicas y cilíndricas para posteriormente enrasarlas según norma EN 12390-2:2009/1M:2015, cumpliendo las características geométricas de las probetas con los parámetros establecidos en la norma EN 12390-1:2013.

Las probetas se mantuvieron en las condiciones de laboratorio durante 24 horas antes de desmoldarse e introducirse en cámara húmeda durante 28 días para su curado, a una temperatura de $20^{\circ} \mathrm{C} \pm 2^{\circ} \mathrm{C}$ y una humedad relativa $\geq 95 \%$.

Después de 28 días en la cámara húmeda, se procedió a la extracción de las probetas de sus moldes, dejándolas a una temperatura ambiente de $22^{\circ} \mathrm{C}$ durante una semana.

Para analizar la conductividad térmica del hormigón en el interior de cada una de las probetas cúbicas, éstas fueron cortadas en dos piezas iguales de $100 \times 100 \times 50 \mathrm{~mm}$ (figura 1) mediante una máquina de corte radial en el laboratorio de la Escuela de Edificación de Madrid.

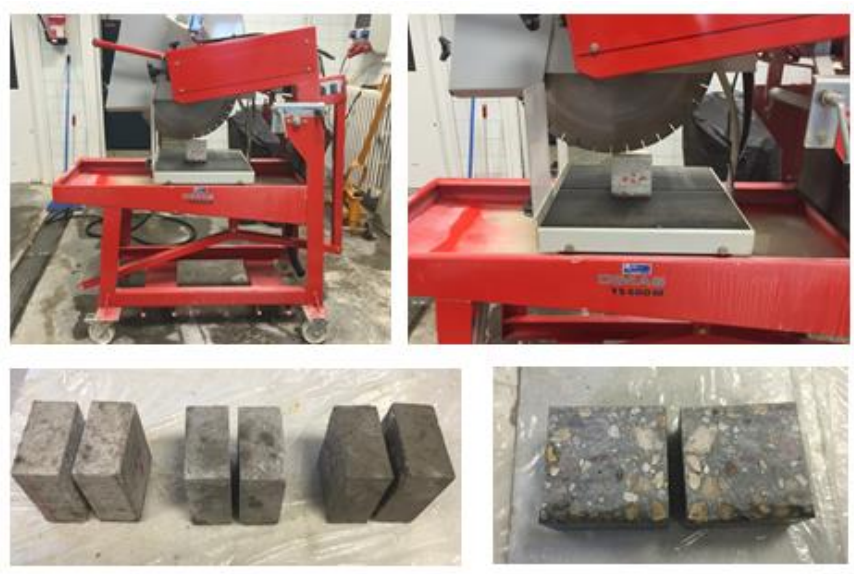

Fig. 1. Proceso de corte de las probetas cúbicas en dos piezas iguales de 100x 100x50mm mediante máquina de corte radial.

Posteriormente las probetas fueron colocadas en el interior de una cámara húmeda hasta alcanzar los grados de humedad del $50 \%$ y $100 \%$, para después medir su conductividad térmica. Cada una de las probetas ensayadas fue sometida al ensayo de conductividad térmica a una temperatura inicial de aproximadamente $20^{\circ} \mathrm{C}$ y una potencia de $1,735 \mathrm{~W}$ durante 3 minutos, con una diferencia de temperaturas máxima de $10{ }^{\circ} \mathrm{C}$ (figura 2).
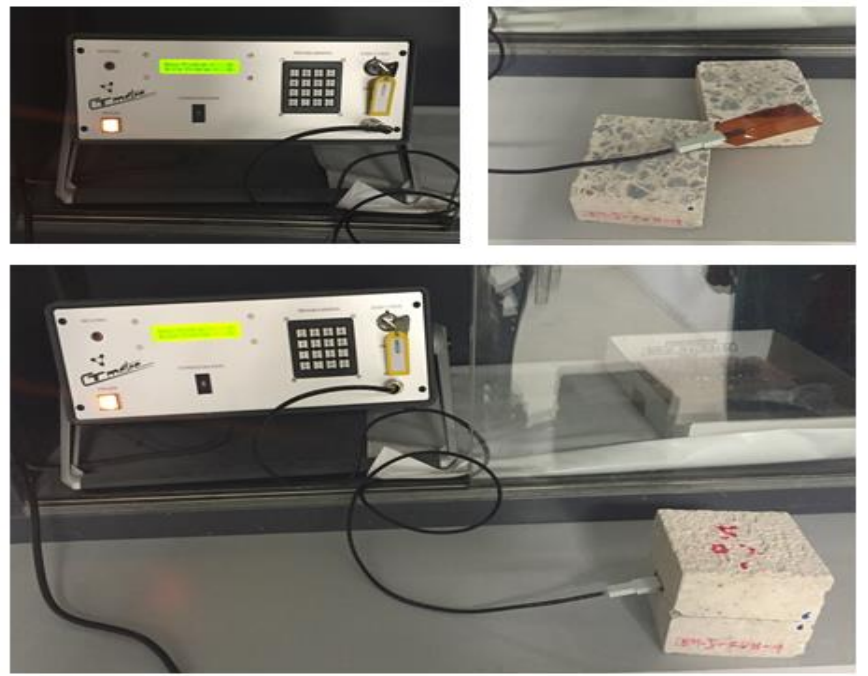

Fig. 2. Proceso de medición de la conductividad térmica del hormigón en probetas cúbicas. 
El comportamiento del hormigón a compresión fue determinado mediante ensayos destructivos siguiendo las especificaciones de la norma UNE-EN 12390-3:2009/AC: 2011.

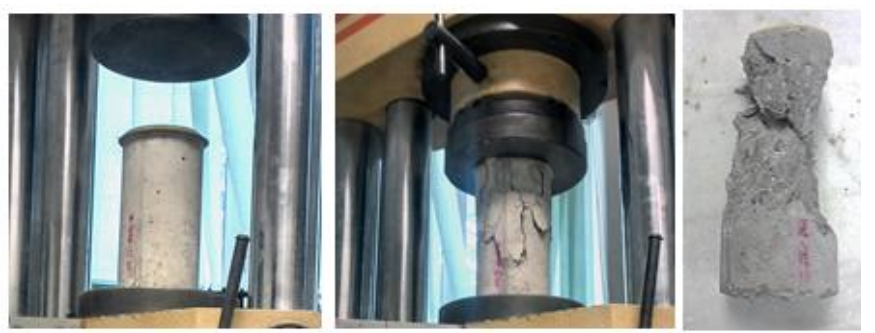

Fig. 3. Rotura de las probetas cilíndricas de hormigón después de haber sido ensayadas a compresión en prensa hidráulica.

Las probetas de hormigón cilíndricas fueron comprimidas en una máquina de ensayo a compresión, hasta registrar la máxima carga alcanzada por la probeta antes de su rotura.

Se llevó a cabo el proceso de refrentado de las tres probetas cilíndricas de cada una de las 6 amasadas, en total 18, según la norma UNE 83506:2004, para posteriormente colocarlas sobre la prensa Universal MIB-60 (figura 3).

\section{RESULTADOS Y ANÁLISIS}

\section{A. Resultados de los ensayos de caracterización térmica}

En los ensayos térmicos realizados sobre probetas cúbicas se obtuvieron 8 valores de conductividad térmica de cada probeta, realizando posteriormente la media aritmética para obtener el valor promedio en $\mathrm{W} /(\mathrm{m} \mathrm{K})$.

En la tabla 2 aparecen los valores medios de conductividad térmica obtenidos en los ensayos realizados sobre las probetas sometidas al $0 \%$ de humedad. La tabla 3 refleja los valores medios de conductividad térmica obtenidos en los ensayos realizados sobre las probetas con $50 \%$ de humedad.

En la tabla 4 se recogen los valores medios de conductividad térmica obtenidos en los ensayos realizados sobre las probetas sometidas al $100 \%$ de humedad.

TABLA II

VALORES MEDIOS DE CONDUCTIVIDAD TÉRMICA DE LAS PROBETAS ENSAYADAS CON UN 0\% DE HUMEDAD RELATIVA

\begin{tabular}{cccc}
\hline \hline Tipo de cemento & $\begin{array}{c}\text { Tipo de } \\
\text { Grava }\end{array}$ & $\begin{array}{c}\text { Grado de } \\
\text { humedad } \\
\text { relativa }\end{array}$ & $\begin{array}{c}\text { Conductivida } \\
\text { térmica } \\
\text { (W/mK) }\end{array}$ \\
\hline CEM II/ B-L 32,5 N & Árido calizo & $0 \%$ & 2,9969 \\
CEM II/ B-L 32,5 N & Árido silíceo & $0 \%$ & 3,8301 \\
CEM I 52,5 N-SR 5 & Árido calizo & $0 \%$ & 3,0600 \\
CEM I 52,5 N-SR 5 & Árido silíceo & $0 \%$ & 4,1364 \\
CEM III/ B 42,5 & Árido calizo & $0 \%$ & 2,4690 \\
CEM III/ B 42,5 & Árido silíceo & $0 \%$ & 3,9311 \\
& & & \\
\hline \hline
\end{tabular}

TABLA III

VALORES MEDIOS DE CONDUCTIVIDAD TÉRMICA DE LAS PROBETAS ENSAYADAS CON UN 50\% DE HUMEDAD RELATIVA

\begin{tabular}{cccc}
\multicolumn{4}{c}{ ENSAYADAS CON UN 50\% DE HUMEDAD RELATIVA } \\
\hline \hline Tipo de cemento & $\begin{array}{c}\text { Tipo de } \\
\text { Grava }\end{array}$ & $\begin{array}{c}\text { Grado de } \\
\text { humedad } \\
\text { relativa }\end{array}$ & $\begin{array}{c}\text { Conductivida } \\
\text { térmica } \\
\text { (W/mK) }\end{array}$ \\
\hline CEM II/ B-L 32,5 N & Árido calizo & $50 \%$ & 2,8845 \\
CEM II/ B-L 32,5 N & Árido silíceo & $50 \%$ & 4,0947 \\
CEM I 52,5 N-SR 5 & Árido calizo & $50 \%$ & 3,1918 \\
CEM I 52,5 N-SR 5 & Árido silíceo & $50 \%$ & 4,1355 \\
CEM III/ B 42,5 & Árido calizo & $50 \%$ & 2,7316 \\
CEM III/ B 42,5 & Árido silíceo & $50 \%$ & 3,6739 \\
& & & \\
\hline \hline
\end{tabular}

TABLA IV

VALORES MEDIOS DE CONDUCTIVIDAD TÉRMICA DE LAS PROBETAS ENSAYADAS CON UN 100\% DE HUMEDAD RELATIVA

\begin{tabular}{cccc}
\hline \hline Tipo de cemento & $\begin{array}{c}\text { Tipo de } \\
\text { Grava }\end{array}$ & $\begin{array}{c}\text { Grado de } \\
\text { humedad } \\
\text { relativa }\end{array}$ & $\begin{array}{c}\text { Conductividad } \\
\text { térmica } \\
\text { (W/mK) }\end{array}$ \\
\hline CEM II/ B-L 32,5 N & Árido calizo & $100 \%$ & 3,2551 \\
CEM II/ B-L 32,5 N & Árido silíceo & $100 \%$ & 5,5188 \\
CEM I 52,5 N-SR 5 & Árido calizo & $100 \%$ & 3,2793 \\
CEM I 52,5 N-SR 5 & Árido silíceo & $100 \%$ & 4,4906 \\
CEM III/ B 42,5 & Árido calizo & $100 \%$ & 2,9509 \\
CEM III/ B 42,5 & Árido silíceo & $100 \%$ & 4,2612 \\
& & & \\
\hline \hline
\end{tabular}

\section{B. Resultados de los ensayos de caracterización mecánica}

La figura 4 muestra los resultados correspondientes a los ensayos de rotura a compresión en las tres probetas cilíndricas analizadas en cada una de las seis amasadas, representados mediante los gráficos de tensión $\sigma\left(\mathrm{N} / \mathrm{mm}^{2}\right)$ - deformación $\varepsilon$ (adimensional).

Los resultados aparecen agrupados en las gráficas en función del tipo de cemento empleado (CEM I, CEM II y CEM III) y el tipo de árido utilizado (calizo o silíceo).

A partir de los ensayos de rotura a compresión se calcularon los valores medios para un análisis más profundo de los resultados. En la tabla 5 aparecen reflejados los valores medios característicos de los ensayos a compresión, tensión máxima $\left(\sigma_{\max }\right)$, deformación máxima $\left(\mathcal{E}_{\max }\right)$, deformación última $\left(\mathcal{E}_{\mathrm{u}}\right)$, densidad de energía de deformación máxima $\left(\mathrm{U}_{\max }\right)$ y densidad de energía de deformación última $\left(\mathrm{U}_{\mathrm{u}}\right)$. La tabla 5 muestra los valores más representativos de los ensayos a compresión agrupados según el tipo de cemento y árido empleado en la elaboración de las probetas. 


\section{DISCUSIÓN}

\section{A. Caracterización térmica}

Los resultados de los ensayos de conductividad térmica se muestran agrupados en la figura 5 permitiendo comprobar que las muestras elaboradas con árido silíceo ofrecieron valores superiores de conductividad térmica a las de las muestras elaboradas con árido calizo, independientemente del tipo de cemento empleado.
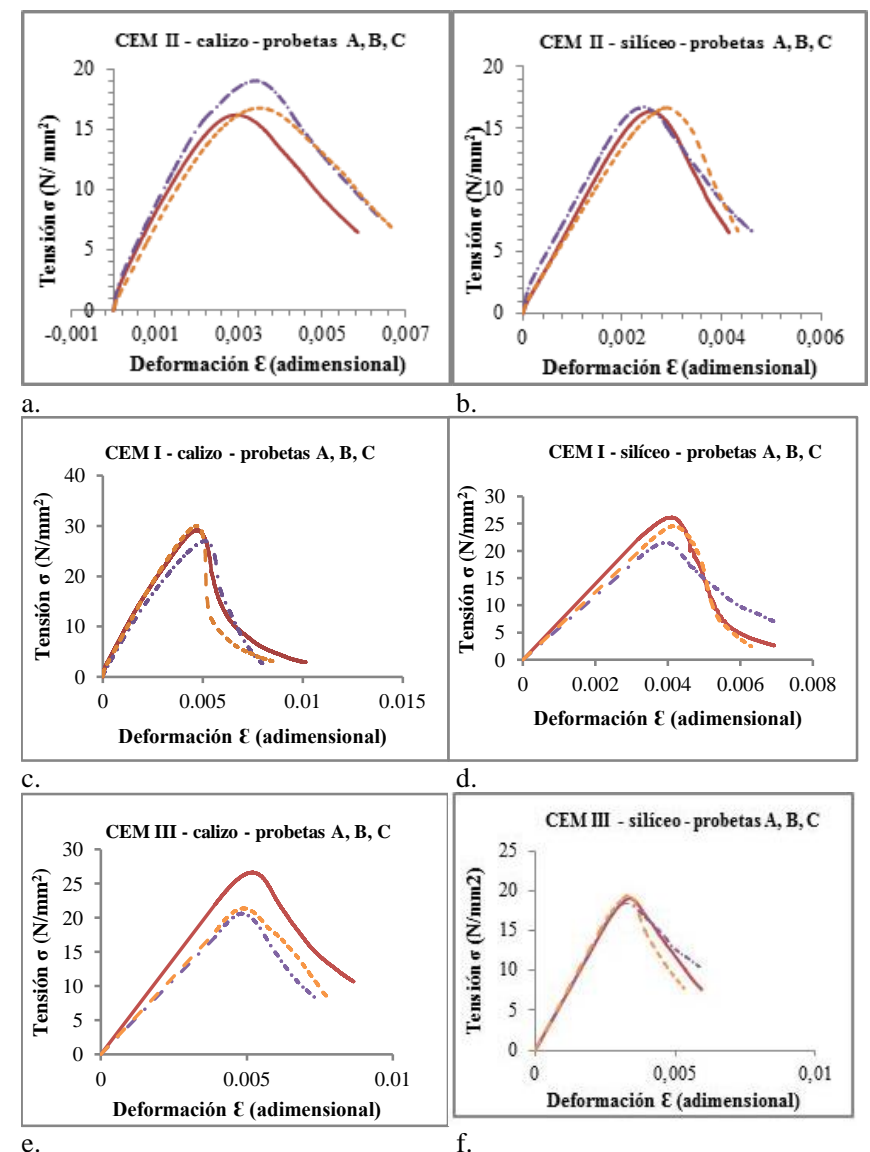

Fig. 4. Gráfica tensión $\sigma\left(\mathrm{N} / \mathrm{mm}^{2}\right)$ deformación $\mathcal{E}$ (adimensional) de los ensayos de compresión de las probetas elaboradas con cemento CEM II y árido calizo (a), cemento CEM II y árido silíceo (b), cemento CEM I y árido calizo (c), cemento CEM I y árido silíceo (d), cemento CEM III y árido calizo (e) y cemento CEM III y árido silíceo (f).

Se comprobó en las muestras de hormigón analizadas la influencia del contenido de humedad. Las probetas con un contenido más elevado de humedad relativa (100\%) ofrecieron valores de conductividad superiores a las de las probetas en estado seco ( $0 \%$ de humedad) o al $50 \%$ de humedad.

En la figura 5 se muestra que los valores de conductividad más altos se dieron en las muestras de hormigón con cemento CEM II, árido silíceo y un $100 \%$ de humedad.

La figura 6 muestra los valores medios de conductividad térmica de todas las probetas cúbicas de hormigón, analizadas en los tres grados de humedad diferentes, $0 \%, 50 \%$ y $100 \%$, independientemente de los tipos de cemento y árido empleados.
TABLA V

VALORES MEDIOS CARACTERÍSTICOS DEL HORMIGÓN EN LOS ENSAYOS A COMPRESIÓN SOBRE PROBETAS CILÍNDRICAS $\left(\sigma_{\max }, \varepsilon_{\max }, \varepsilon_{\mathrm{u}}, \mathrm{U}_{\max }, \mathrm{U}_{\mathrm{u}}\right.$ y Módulo

\begin{tabular}{|c|c|c|c|c|c|c|c|}
\hline $\begin{array}{l}\text { Tipo de } \\
\text { cemento }\end{array}$ & $\begin{array}{c}\text { Tipo } \\
\text { de } \\
\text { Grava }\end{array}$ & $\begin{array}{c}\sigma_{\max } \\
(\mathrm{N} / \\
\left.\mathrm{mm}^{2}\right)\end{array}$ & $\varepsilon_{\max }$ & $\varepsilon_{\mathrm{u}}$ & $\begin{array}{c}\mathrm{U}_{\max }(\mathrm{N} / \\
\left.\mathrm{mm}^{2}\right)\end{array}$ & $\begin{array}{c}\mathrm{U}_{\mathrm{u}}(\mathrm{N} / \\
\left.\mathrm{mm}^{2}\right)\end{array}$ & $\begin{array}{l}\text { Módulo } \\
\text { secante } \\
\left(\mathrm{N} / \mathrm{mm}^{2}\right)\end{array}$ \\
\hline $\begin{array}{c}\text { CEM II/ } \\
\text { B-L 32,5 } \\
\mathrm{N}\end{array}$ & 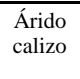 & 17,32 & 0,0033 & 0,0057 & 0,0368 & 0,0741 & 8140,43 \\
\hline $\begin{array}{c}\text { CEM II/ } \\
\text { B-L 32,5 } \\
\text { N }\end{array}$ & $\begin{array}{l}\text { Árido } \\
\text { silíceo }\end{array}$ & 16,59 & 0,0026 & 0,0043 & 0,0220 & 0,0414 & 7940,99 \\
\hline $\begin{array}{c}\text { CEM I } \\
52,5 \mathrm{~N}- \\
\text { SR } 5\end{array}$ & $\begin{array}{l}\text { Árido } \\
\text { calizo }\end{array}$ & 28,74 & 0,0048 & 0,0089 & 0,0796 & 0,1232 & 7822,53 \\
\hline $\begin{array}{c}\text { CEM I } \\
52,5 \mathrm{~N}- \\
\text { SR } 5\end{array}$ & $\begin{array}{l}\text { Árido } \\
\text { silíceo }\end{array}$ & 24,07 & 0,0035 & 0,0075 & 0,0492 & 0,0875 & 8336,93 \\
\hline $\begin{array}{l}\text { CEM III/ } \\
\text { B 42,5 }\end{array}$ & $\begin{array}{l}\text { Árido } \\
\text { calizo }\end{array}$ & 22,88 & 0,0038 & 0,0068 & 0,0496 & 0,0987 & 7096,91 \\
\hline $\begin{array}{l}\text { CEM III/ } \\
\text { B } 42,5\end{array}$ & $\begin{array}{l}\text { Árido } \\
\text { silíceo }\end{array}$ & 19,00 & 0,0030 & 0,0059 & 0,0334 & 0,0708 & 8000,54 \\
\hline
\end{tabular}

Las probetas con un contenido de humedad del $100 \%$ mostraron valores de conductividad térmica con una media de $3,959 \mathrm{~W} / \mathrm{m} \mathrm{K}$. Las muestras con un contenido de humedad del $50 \%$ ofrecieron medias de conductividad de $3,452 \mathrm{~W} / \mathrm{m} \mathrm{K}$, mientras que las probetas en estado seco mostraron valores inferiores de conductividad, con valores medios de $3,403 \mathrm{~W} / \mathrm{m}$ $\mathrm{K}$.

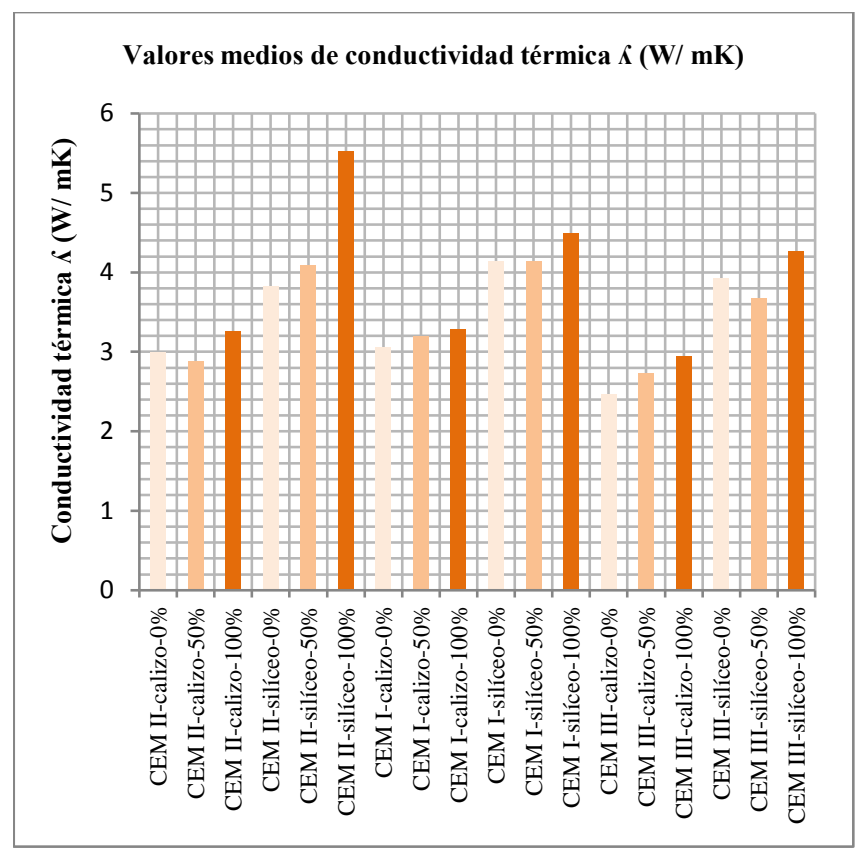

Fig. 5. Valores medios de conductividad térmica $\Lambda(\mathrm{W} / \mathrm{m} \mathrm{K})$ obtenidos de los ensayos realizados sobre probetas cúbicas de hormigón con humedad relativa del $0 \%, 50 \%$ y $100 \%$.

Se observó un incremento de los valores de conductividad térmica del $14 \%$ en las probetas en estado saturado con respecto de las probetas analizadas en estado seco.

Los resultados obtenidos siguen la misma tendencia que otras investigaciones llevadas a cabo sobre la conductividad térmica del hormigón y sus componentes, como el de Fargueta 


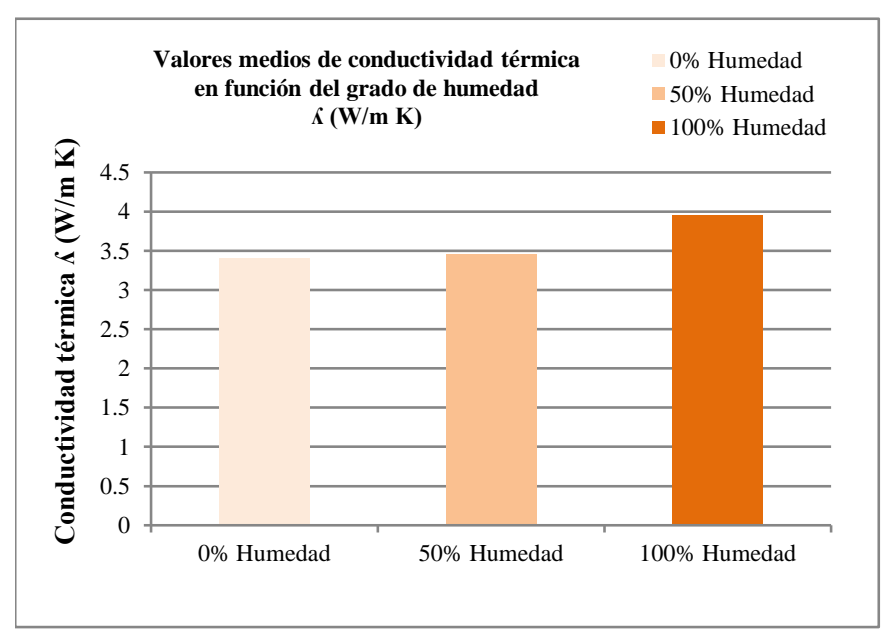

Fig. 6. Valores medios de conductividad térmica $\kappa(\mathrm{W} / \mathrm{m} \mathrm{K})$ de las probetas cúbicas de hormigón, en función de los tres grados de humedad relativa, $0 \%$, $50 \%$ y $100 \%$.

(Fargueta \& Benavent, 2010) en los que se concluyó que el árido silíceo presenta valores de conductividad más elevados que el calizo.

En otros estudios se concluyó que la porosidad en el hormigón provoca la disminución de los valores de conductividad térmica, siendo mayor esta en los hormigones secos que en los saturados (Fernandez, 1999). En estudios como el de Naus (Naus, 2010), se obtuvieron resultados en referencia a la conductividad térmica del hormigón observando que los factores primarios que la determinan son su contenido de humedad, el volumen de poro y su distribución, y la cantidad y tipo de árido. Esto se debe en parte a que el hormigón elaborado con cemento de tipo CEM I 52,5 N-SR 5 pertenece a una clase superior a 32,5, al contrario que el cemento CEM II y CEM III empleado en el resto de amasadas.

\section{B. Caracterización mecánica}

La figura 7 permite comparar los valores de tensióndeformación obtenidos en los ensayos destructivos a compresión sobre las probetas cilíndricas analizadas.

La figura 7 a muestra que las probetas elaboradas con cemento CEM II y árido calizo presentaron una resistencia a compresión mayor que las elaboradas con árido silíceo, llegando a un valor de tensión máxima $\left(\sigma_{\max }\right)$ de 17,32 N/mm2 y deformación máxima $\left(\mathcal{E}_{\max }\right)$ de 0,0033 .

En la figura $7 \mathrm{~b}$ se observan los valores de tensión deformación para las probetas elaboradas con cemento CEM I y árido calizo y silíceo. Las muestras con árido calizo ofrecieron valores de tensión máxima $\left(\sigma_{\max }\right)$ de $28,74 \mathrm{~N} / \mathrm{mm} 2$ y de deformación máxima $\left(\varepsilon_{\max }\right)$ de 0,0048 , superiores a los alcanzados por las muestras elaboradas con árido silíceo.

En la figura 7c se muestran los valores medios de tensión y deformación de los ensayos a compresión de probetas cilíndricas elaboradas con cemento CEM III y árido calizo y silíceo respectivamente. Las probetas elaboradas con árido calizo alcanzaron valores superiores de resistencia a compresión con valores de tensión máxima $\left(\sigma_{\max }\right) 22,88 \mathrm{~N} /$ mm2 y deformación máxima $\left(\mathcal{E}_{\max }\right) 0,0038$.

Los resultados obtenidos coinciden con las de otras investigaciones. En el estudio de Alaejos (Alaejos \& Fernández, 1994) se analizó la influencia de los áridos en la resistencia a compresión del hormigón, concluyendo que el árido calizo ofrece resistencias superiores a las de otros áridos en el hormigón.

En otros estudios se analizó la influencia del tipo de cemento empleado en la elaboración de hormigones determinando que su influencia es menor que la de otros componentes, como pueden ser los áridos (Neville, 1982).

La influencia del cemento empleado en el hormigón se ve condicionada por su grado de hidratación, ya que tiene un efecto directo sobre la porosidad, y por tanto, sobre su resistencia. El cemento portland de tipo III, según la norma ASTM (American Society for Testing and Materials), hidrata más rápidamente que otros cementos a edades tempranas, y tiene una mayor resistencia como consecuencia de su mayor porosidad (Kumar Mehta \& J.M. Monteiro, 2013).

\section{CONCLuSIONES}

\section{A. Conclusiones a los resultados de caracterización térmica.}

En los ensayos de conductividad térmica se obtuvieron valores de conductividad más elevados en las muestras de hormigón elaboradas con árido silíceo, corroborando estudios de otros autores, que apuntan que el árido silíceo presenta una conductividad térmica más elevada que el árido calizo (Fargueta, et al, 2010), (Savva, Manita \& Sideris, 2005), (Arioz, 2007).

Se observó un incremento de los valores de conductividad térmica del $14 \%$ en las probetas que se encontraban en estado saturado ( $100 \%$ de humedad) con respecto de los obtenidos en las muestras al $0 \%$ de humedad. En la misma línea, existen estudios anteriores que apuntan que hormigones en estado saturado y con baja porosidad presentan conductividades térmicas más elevadas que hormigones con muestras en estado seco (Capote, Albear \& Crespo, 2011).

Contrariamente a lo que se esperaba, se obtuvieron valores de conductividad más elevados en las muestras de hormigón elaborado con cemento de tipo CEM II.

Las muestras de hormigón elaboradas con cemento CEM III, de origen siderúrgico, al contrario de lo que se esperaba, presentaron conductividades inferiores a las de muestras de hormigón elaboradas con cemento de tipo CEM II y CEM I.

Con el fin de conseguir valores de conductividad térmica mayores en el hormigón, es recomendable elaborar hormigones con una baja relación agua/cemento, que presenten valores de porosidad bajos. 


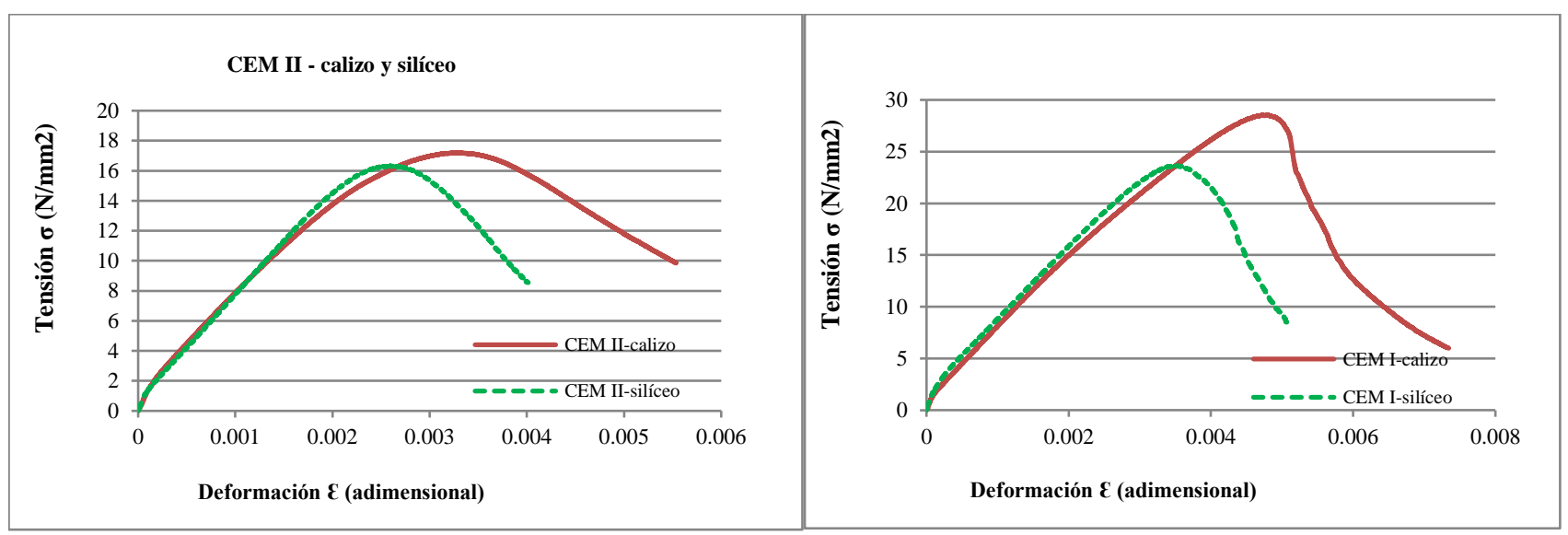

(a)

(b)

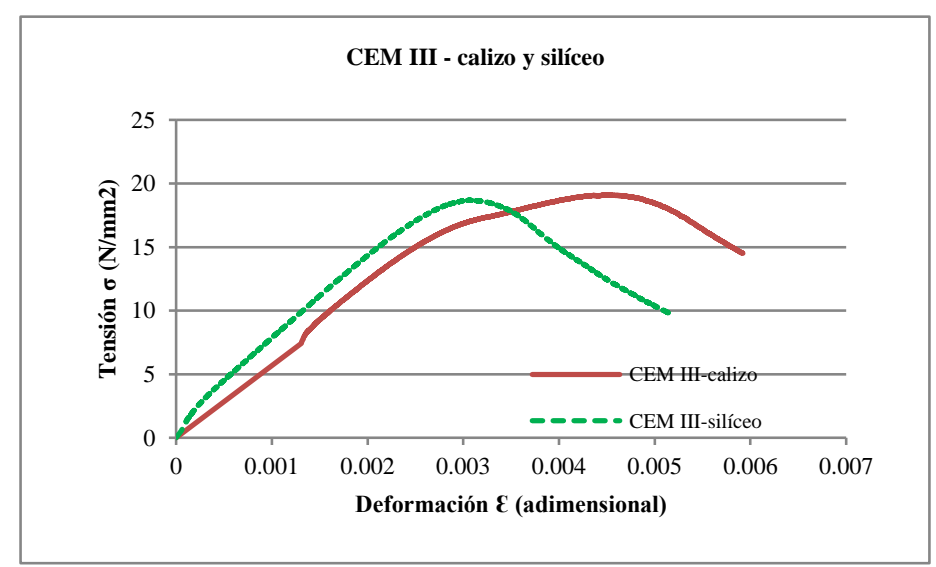

(c)

Fig. 7. Diagramas tensión-deformación obtenidos de los ensayos destructivos a compresión sobre probetas cilíndricas elaboradas con cemento CEM II y árido calizo y silíceo (a), CEM I y árido calizo y silíceo (b) y CEM III combinadas con árido calizo y silíceo (c).

Podría concluirse que el árido silíceo es más recomendable en la elaboración de hormigones para estructuras termoactivas, elaborados con los cementos estudiados en este trabajo. Esta ventaja es superior cuando el hormigón está prácticamente saturado.

\section{B. Conclusiones a los resultados de caracterización mecánica.}

De los ensayos a compresión realizados se obtuvieron los valores de resistencia más altos en las probetas realizadas con cemento CEM I y árido calizo.

Las probetas elaboradas con cemento CEM II y árido silíceo ofrecieron los valores de resistencia más bajos con respecto a los obtenidos en las amasadas elaboradas con cemento de tipo I y III, independientemente del tipo de árido empleado en el hormigón para la elaboración de las probetas.

En todas las amasadas, independientemente del tipo de cemento empleado, se obtuvieron valores de resistencia superiores en las probetas con árido de origen calizo.

Se observó una diferencia del $42,8 \%$ en los valores de resistencia máxima $\left(\sigma_{\max }\right)$ obtenidos en los ensayos realizados con probetas de hormigón con cemento CEM I y árido calizo (valores máximos), con respecto de las elaboradas con cemento CEM II y árido silíceo.

Se obtuvieron valores de deformación máxima $\left(\varepsilon_{\max }\right)$, en los ensayos realizados sobre probetas elaboradas con cemento CEM I y árido silíceo, un 45,84\% inferiores a los obtenidos en las probetas de hormigón elaborado con cemento CEM II y árido de origen calizo.

Los resultados obtenidos revelaron la gran influencia que ejerce el tipo de árido sobre los valores de resistencia del hormigón ante los ensayos a compresión.

Las muestras de hormigón elaboradas con cemento CEM II y árido calizo ofrecieron valores de conductividad más elevados que los de las otras muestras analizadas, por lo que podría recomendarse su uso en estructuras termoactivas siempre que alcancen los requerimientos estructurales exigidos.

\section{REFERENCIAS}

Alaejos, M.P., Fernández, M. (1994). Selección de materiales para la fabricación de hormigones de alta resistencia. Materiales de la Construcción Vol. 44 N 235.

Arioz, O. (2007). Effects of elevated temperature on properties of concrete. Fire Safety Journal 42 (2007) 516-552. 
Brandl, H. (2006). Energy foundations and other thermo-active ground structures. Geotechnique 56: 3-44.

Capote, J.A., Albear, M.D., Crespo, J. (2011). Análisis experimental de hormigones expuestos al fuego. Evaluación de variables hidro-térmicas. Ingeniería e Industria. Rev. 1 del 30/dic/2011.

Fargueta, F., Benavent, J., (2010). Estudio experimental de los efectos de la temperatura sobre la resistencia del homigón. II Congreso Nacional de Investigación en Edificación. UPM. Madrid. España.

Fernandez Cánovasç. M. (1999). Hormigón. Colegio de Ingenieros de Caminos, Canales y Puertos. Madrid, España. I.S.B.N.: 84-7493-125-8

Fu, Y., Li, L. (2010). Study on mechanism of thermal spalling in concrete exposed to elevated temperatures. Materials and Structures 44: 361-376.

Kumar Mehta, P., J.M. Monteiro, P., (2013) Concrete: Microestructure, Properties and Materials. Editorial McGraw-Hill. ISBN-13:978-0071797870.

Lehmann, B., Dorer, V., Koschenz, M. (2007). Application range of thermally activated building system tabs. Energy and Buildings 39 Issue 5: 593-598.

Naciones Unidas (1992). “Convención Marco de las Naciones Unidas sobre el cambio climático".

Naus, D.J. (2010). A compilation of Elevated Temperature Concrete Material Property Data and Information for Use in Assesments of Nuclear Power Plant Reinforced Concrete Structures" U.S. NRC.

Neville, A.M. (1982). Properties of concrete. Editorial Pitman. ISBN: 0273016423.

Ortega, I. (2014). Guía sobre estructuras termoactivas y sistemas inerciales en la climatización de edificios. Capítulo 1: Soluciones de Climatización Invisibles. Fenercom.

RC-16. (2016). Instrucción para la recepción de cementos. Secretaría General Técnica, Ministerio de Fomento, Madrid, España.
Savva, A., Manita, P., Sideris, K.K., (2005). Influence of elevated temperatures on the mechanical properties of blended cement concretes prepared with limestone and siliceous aggregates. Cement and Concrete Composites 27 (2005) 239-248.

Svanholm, G., (1980). Leightweight concrete. The concrete society. Thermal conductivity as quality parameter. International Congress on Lightweight Concrete. ISBN: 086095-856-6.

Torio, H., Scmidt, D. (2011). Exergy Assessment Guidebook for the Built Environment: Annex 49, Summary report.

UNE-EN 12620:2003+A1:2009. (2003). Áridos para hormigón. CEN/TC/154 Áridos (2009).

UNE-EN 12390-2:2009/1M:2015. (2015). Ensayos de hormigón endurecido. Parte 2: Fabricación y curado de probetas para ensayos de resistencia.

UNE-EN 12390-1:2013. (2014). Ensayos de hormigón endurecido. Parte 1: Forma, dimensiones y otras características de las probetas y moldes.

UNE-EN 197-1:2011. (2011). Cemento. Parte 1: Composición, especificaciones y criterios de conformidad de los cementos comunes. CEN/TC 51 "Cement and building limes", 2011.

UNE-EN 12390-3:2009/AC:2011. (2011). Ensayos de hormigón endurecido. Parte 3: Determinación de la resistencia a compresión de probetas.

UNE 83506:2004. (2004). Hormigones con fibras. Refrentado de probetas con mortero de azufre.

Youssef, M.A., Moftah, M. (2007). General stress-strain relationship for concrete at elevated temperatures. Engineering Structures 29 (2007) 2618-2634.

Zoldners, N.G. (1973). Thermal properties of concrete under sustained elevated temperatures. Temperature and concrete. American Concrete Institute. SP-25 33-50. 